\title{
Buddy System: Available, Consistent, Durable Web Service Transactions
}

\author{
Aspen Olmsted \\ Department of Computer Science \\ College of Charleston \\ Charleston, SC 29401
}

\author{
Csilla Farkas \\ Center for Information Assurance Engineering \\ Department of Computer Science and Engineering \\ University of South Carolina, Columbia, SC 29208
}

\begin{abstract}
In this paper we investigate the problem of providing consistency, availability and durability for web service transactions. We propose an approach that matches the availability of the popular lazy replica update propagation method while increases the durability and consistency. Our replica update propagation method is called the Buddy System, which requires that updates are preserved synchronously in two replicas. Our first implementation schedules fine-grained WS transactions. In these transactions each activity is a low level database operation. Later we consider each transaction as a black box, with only the corresponding metadata, expressed as UML specifications, as transaction semantics. We refer to these WS transactions as coarse-grained WS transactions. The Buddy System is able to handle these course grained WS transactions, using UML stereotypes that allow scheduling semantics to be embedded into the design model. We show that our approach guarantees one-copy serializability, matches the performance of the lazy update propagation methods, and increases durability in the presence of hardware failures.
\end{abstract}

\section{Introduction}

Modern web based transaction systems need to support many concurrent clients simultaneously consuming a limited quantity of resources. These applications are often developed using a Service Oriented Architecture (SOA). SOA supports the composition of multiple web services (WSs) to perform complex business processes. One of the important aspects for SOA applications is to provide a high-level of concurrency; we can think of the measure of the concurrency as the availability of the service to all clients requesting services. A common way to increase the availability is through the replication of the services and their corresponding resources. Unfortunately consistency and durability are often sacrificed to achieve this availability. The CAP theory $[1,2]$, stating that distributed database designers can achieve at most two of the properties: consistency (C), availability (A), and partition tolerance $(\mathrm{P})$ has influenced distributed database design in a way that often causes the designer to relax the consistency requirements.
The standard architecture used to increase the availability of a system is through a web service (WS) farm (Figure 1). The WS farm may host multiple replicas of the services and their resources. Service requests are distributed among the replicas within a WS farm to ensure a high availability. Usually, a WS farm is placed behind a dispatcher. Clients send service requests to the dispatcher, and the dispatcher distributes the requests to one of the redundant services. In a simple architecture, the redundant web servers share a single database, so all web service replicas have access to the same data. However, depending on the replica update propagation method deployed, mutual consistency of the replicated database and satisfaction of integrity constraints are not always guaranteed.

This paper addresses the issues related to increasing service and data availability while still guaranteeing durability and consistency of replicated databases in the context of SOA. Our approach is based on requiring that two of the replicated services must update their corresponding databases simultaneously, thus reducing the risk of data loss due to hardware failures. The selection of the web service pair, called buddies, supports consistency. We study the problem in two contexts. First, we model the web services as database transactions, requesting read and write access to the data items. Second, we model the services as black boxes, when only the input and output parameters of the services are known. We represent this information using the UML stereotype construct. For each context, we develop algorithms for buddy selection and update propagation. We show that our solutions guarantee one-copy serializability. Moreover, we show that our approach ensures that data is distributed in a way that provides enforcement of referential integrity, protects against data loss due to hardware failures, and provides high levels of availability. We present our empirical results for both contexts.

The organization of the paper is as follows. Section 2 lays out an example transaction. Section 3 addresses the problem using fine-grained web services. Section 4 identifies a typical user-defined constraint that when enforced reduces availability. We provide a solution to enforce this constraint while maintaining high availability. Section 5 looks at the problem at a coarse-grained level where the services are abstract functions. Section 6 describes the related work and the limitations of current 
methods. We conclude and discuss future work in Section 7.

\section{Example transaction}

Consider a Ticket Reservation System (TRS). TRS uses web services to provide a variety of functionalities to the clients.

For example, clients may check ticket availability or request a ticket reservation. Upon receiving a client's request, the Availability Check service searches for ticket availability based on the client's requirements and preferences. For example, the client may be looking for aisle seats between May 1st and May 3rd, 2013 to a Mozart concert in Charleston, SC. The service returns a list of ticket rates available with additional properties, such as seating layout, acoustics, etc. The Availability Check service relies on replicated databases to improve the processing time.

Using lazy-replication architectures, Availability Check service may retrieve outdated data and, therefore, provide an incorrect response to the client. If strict replication propagation is supported, this request may time-out due to process contention if the request is concurrent with other availability requests.

The Ticket Reservation service reserves a ticket for the client, based on the requirements and preferences given by the client. For example, the service may book two main floor tickets on May 3rd, 2013 to the Mozart concert in Charleston, SC at a rate of \$99/ticket. Using lazy update propagation, the update is performed in an efficient manner but may lead to lost data (e.g., if the server where the update transaction runs crashes after the transaction committed but before the other replicas are updated) and temporary data inconsistency (e.g., if referential integrity spans masters). Strict update propagation guarantees consistency and durability but reduces the availability.

\section{Proposed system}

Our proposed system addresses three problems: decrease the risk of losing committed transactional data in case of a site failure, increase consistency of transactions, and increase availability of read requests. The three main components of our proposed system are: 1) Synchronous Transactional Buddy System, 2) Version Master-Slave Lazy Replication, and 3) Serializable Snapshot Isolation Schedule.

We adopt the WS-Farm architecture (Figure 1). Transactions arrive to the dispatcher at the OSI TCP/IP level 7. This allows the dispatcher to use application specific data for transaction distribution and buddy selection. The dispatcher receives the requests from clients and distributes them to the WS clusters. Each WS cluster contains a load balancer, a single database, and replicated services. The load balancer receives the service requests from the dispatcher and distributes them among the service replicas. Within a WS cluster, each service shares the same database. Database updates among the clusters are propagated using lazy replication propagation.

\subsection{Preliminaries}

A Web Service Farm is set of Web Service Clusters $\mathrm{WSF}=\left\{\mathrm{WSC}_{1}, \ldots, \mathrm{WSC}_{\mathrm{n}}\right\}$. A single dispatcher receives requests from clients and distributes these requests to WS-Clusters.

A WS-Cluster is a group of WS-Replicas that share a single data store and a load balancer. Each WSCluster (WSC) is represented as a three tuple WSC = (WS, HW, DB), where $\mathrm{HW}=\left\{\mathrm{hw}_{1}, \ldots, \mathrm{hw}_{\mathrm{n}}\right\}$ is a set of common, off-the-shelf (COTS) hardware devices. DB is a database. In this work, we consider relational databases. The load balancer distributes load to the service replicas in the cluster.

A set of WS-Replicas, WS $=\left\{\mathrm{ws}_{1}, \ldots, \mathrm{ws}_{\mathrm{n}}\right\}$ a set of replicated web services with the same functionality. WS-Replica Buddies or simply buddies are $\mathrm{ws}_{\mathrm{i}}$ and $\mathrm{ws}_{\mathrm{j}}$, such that $\mathrm{ws}_{\mathrm{i}}$ and $\mathrm{ws}_{\mathrm{j}}$ belong to two different WS clusters. .

A Database Transaction is a partial order of read and write operations on data items, and a single abort or commit. We denote a transaction $\mathrm{T}$ as follows, $\mathrm{T}=$ $\{\leq, r[d], w[d] \mid d \in D B, c / a\}$. The read-set of a transaction $T$ denotes all the data items $d \in D B$ such that there is a $r[d] \in T$. The write-set of a transaction $T$ denotes the data items $d \in D B$ such that there is a $\mathrm{w}[\mathrm{d}] \in \mathrm{T}$.

Data item version denotes a data value and its version number. Given a database $\mathrm{DB}=\left\{\mathrm{d}_{1}, \ldots, \mathrm{d}_{\mathrm{n}}\right\}$ each data item $d_{i}(i=1, \ldots, n)$ is associated with a single version number $\mathrm{v}_{\mathrm{n}}$. Initially each data item's version number is 0 . Version numbers are incremented by one when a data item is updated by a transaction. For clarity we model the database as pairs of data item and version numbers, that is $\mathrm{DB}=$ $\left\{\left(\mathrm{d}_{1}, \mathrm{v}_{1}\right), \ldots,\left(\mathrm{d}_{\mathrm{n}}, \mathrm{v}_{\mathrm{n}}\right)\right\}$.

Each database is associated with a version number. Given a database DB and the data items $\left\{\left(\left(\mathrm{d}_{1}, \mathrm{v}_{1}\right)\right.\right.$, $\left.\left(d_{2}, v_{2}\right), \ldots,\left(d_{n}, v_{n}\right)\right\}$ in $D B$, we say the version numbers of DB is the vector $\left\langle\mathrm{v}_{1}, \ldots, \mathrm{v}_{\mathrm{n}}\right\rangle$.

DB-Replicas, denoted as DBR $=\left\{\mathrm{dbr}_{1}, \ldots, \mathrm{dbr}_{\mathrm{n}}\right\}$, are databases originating from the same database (i.e.,version $\left.<0_{1}, \ldots, 0_{n}\right\rangle$ ). Given two replicas, $\mathrm{dbr}_{1}$ and $\mathrm{dbr}_{\mathrm{j}}$, they may or may not have the same version number.

\subsection{Buddy system}

Lazy update propagation is vulnerable for loss of updates in the presence of a database server failure. The window of vulnerability for this loss is 


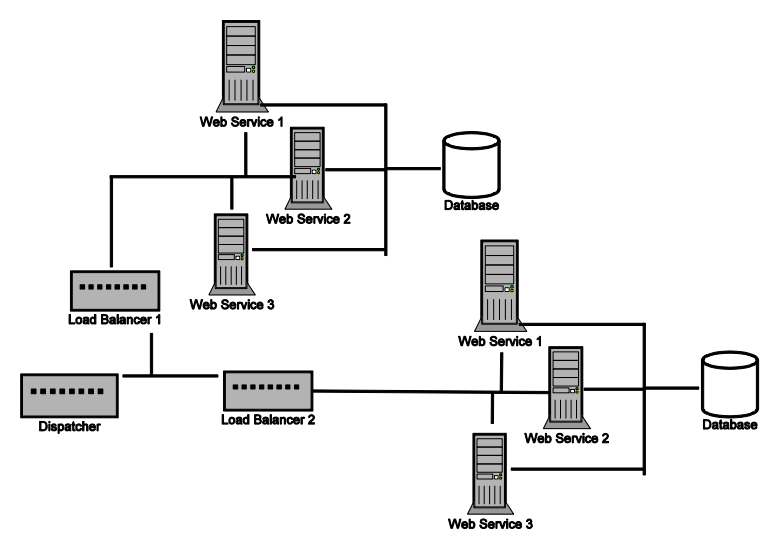

Figure 1. WS Farm with Buddy System

after the transaction has committed but before the replica updates are initiated. To guarantee data persistence even in the presence of hardware failures we propose to form strict replication between pairs of replica clusters "buddies." Our aim is to ensure that at least one of the replicas in addition to the primary replica is updated and, therefore, preserves the updates.

Figure 1 shows a WS farm (WSF) architecture where each cluster (WSC) has a load balancer (LB). After receiving a transaction, the dispatcher picks the two clusters to form the buddy-system. The selection is based on versioning history. The primary buddy (b1) receives the transaction along with its buddy's (b2) IP address. The primary buddy (b1) becomes the coordinator in a simplified commit protocol between the two buddies. Both buddies perform the transaction and commit or abort together. The dispatcher maintains metadata about the freshness of data items in the different clusters. The dispatcher increments the version number for each data item after is has been modified. Any two service providers in two different clusters with the latest version of the requested data items can be selected as a buddy. Note, that the databases (DBR) maintained by the two clusters must have the same version of the requested data items but may be different for the other data items.

\subsection{Dispatcher data structures}

The dispatcher maintains a version table for every data object modified by the web services. When a request is received for a service, the dispatcher ensures that the request is delivered to the appropriate cluster. If the request is read-only, the primary buddy (b1) must have the latest version of all committed objects in the request. If the request includes writes, the dispatcher determines whether there is any uncommitted transaction accessing the requested data objects. If there is such a transaction, then the request is sent to the web service cluster where the active transaction is being executed. If the dispatcher cannot find a cluster with the latest version no suitable cluster can be found due to the distribution of the requested object then the service request is queued until the currently active transactions complete or the updates are propagated. To avoid snapshot isolation anomalies, we address blind writes and analyze the read log to determine if an anomaly could take place. Fekete et al. [3] documented anomalies that must be avoided to turn a snap shot isolation schedule into a serialized schedule. We incorporate these requirements to support serializable execution. The dispatcher maintains the following data structures for processing the algorithms:

- Cluster List - contains the names of the clusters (WSC) and their IP addresses.

- Objects Version Table - contains the name of the data objects and their version numbers,

corresponding to the completed and in-progress transactions.

- Cluster Object Table - contains the cluster names, stored objects, and the version number of the objects at that cluster.

Table 1.a, b, and $\mathrm{c}$ show examples of the above tables. The Cluster List Table shows the three clusters 1, 2, and 3, the Object Version Table indicates that data objects B and C are not currently being updated, but object $\mathrm{A}$ has two update operations still in-progress at clusters 1 and 2 .

\subsection{Dispatcher service request algorithm}

The dispatcher service request algorithm (Algorithm 1) is executed by the dispatcher for every incoming request containing write operations. The goal of the service request algorithm is to find a pair of buddies that have the correct version for the incoming request. If a pair cannot be found then the request is delayed for later processing.

For read only requests, the dispatcher executes a simplified version of the algorithm. This version only requires a single cluster, containing the latest committed values of the requested objects, to respond to the request. Due to space limitation, we omitted the read-only algorithm in this work.

Theorem 1: The Dispatcher Service Request Algorithm (Algorithm 1) guarantees one-copy serializability.

Proof. Our proof is based on the following claim: Let $\mathrm{H}$ be a history over a set of transactions $\mathrm{T}$, such that each transaction $\mathrm{Ti} ; \mathrm{i}=1, \ldots, \mathrm{n}$ is made up of a set of read $\mathrm{R}_{\mathrm{i}}(\mathrm{d})$ and write $\mathrm{W}_{\mathrm{i}}(\mathrm{d})$ operations on data items $\mathrm{d}$. $\mathrm{H}$ is one-copy serializable if the following three conditions hold:

1. Each request (transaction) is an atomic transaction 
2. Concurrent writes on the same data item are sent to the same cluster (WSC), and

3. Each cluster guarantees serializable transaction history on its local database.

To show that the claim holds, assume, by contradiction that $\mathrm{H}$ is not one-copy serializable. Then, there must exists a cycle among the committed transactions in the serialization graph of $\mathrm{H}$. Let $\mathrm{T}_{\mathrm{i}}$ and $T_{j}$ be the two transactions responsible for the cycle. The serialization graph cannot contain a cycle for the three potential scenarios or these transactions. The three scenarios are:

Read Set/Write Set overlap - The transaction containing the read set will be sent to any cluster containing the latest committed version of the elements of the read set, effectively scheduling the read set transaction before the write set transaction.

Write Set/Write Set overlap - Both transactions will be sent to the same cluster. The database management system guarantees serializable execution at that cluster, and, therefore, one-copy serializability.

Read Set/Read Set overlaps - There is no conflict.

Transaction processing between the primary and secondary buddies is synchronous and both will either commit or abort. After a committed update operation on a data object, the dispatcher updates the Object Version Table to represent the current version numbers. When a primary buddy or any lazy update cluster completes a transaction, it sends a version update request to the dispatcher. The dispatcher updates the latest completed version value for these clusters. After the version is updated all requests in the queue are reprocessed in hopes that the dispatcher can now find a pair of buddies with the correct versions.

\subsection{Load Balancing}

Algorithm 1 chooses the first available cluster for read only requests and the first available pair of clusters for requests containing write operations. This may create unbalanced use of the WS clusters. The selection can be improved by decorating the Cluster List Table (Table 1.a) with attributes to represent system properties (e.g., processing power, available applications, process wait-time, etc.) and network-related information (e.g., link properties, hop-distances, etc.). These attributes can be used to influence buddy selection, e.g., provide load balancing among the clusters or select clusters based on their geographical location and the reliability of the communication network. This improved buddy selection is not presented in this work.

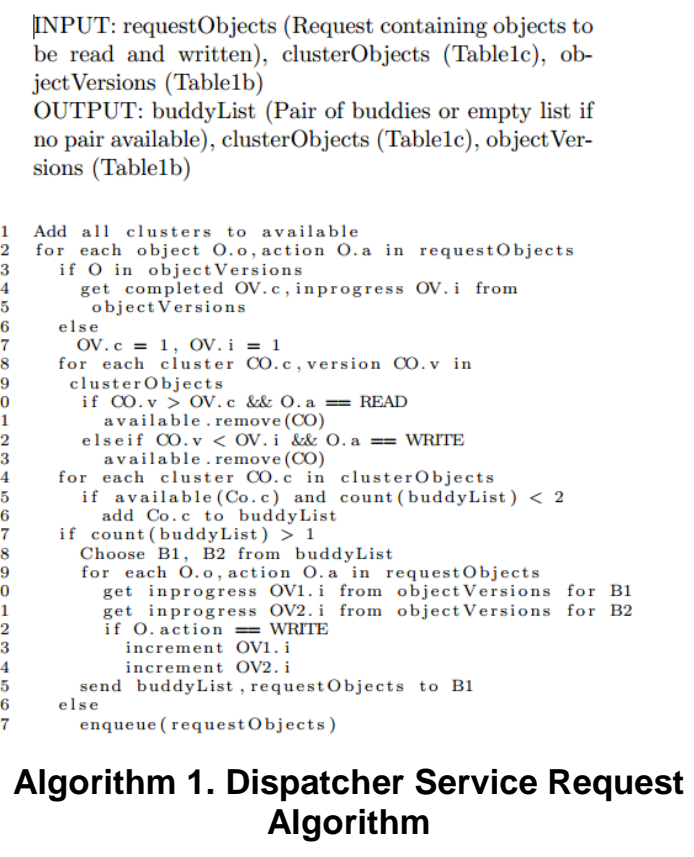

\subsection{Analysis of the buddy system}

In this section we evaluate the performance of our system in high-volume scenarios, and compare our approach with eager and lazy replica update propagation in the presence of hardware failures.

Performance analysis in high volume scenarios: Some of the transactions involve large volumes of data items of the same type. For example, multiple concert tickets have the same characteristics but different row and seat numbers. Performance characteristics of transactions involving a large number of similar objects can be improved by considering the type of resource consumption. We distinguish between three types of consumption patterns:

Anonymous Item Consumption when each object is interchangeable, for example all seats for general admission for a concert. In this case, the buddy system does not improve latency over simple masterslave replication since all concurrent resources requests need to be sent to the same buddy pair.

Attribute Item Consumption when each client's request has filters on the attributes, such as mainfloor or balcony seating for a concert. The buddy system improves latency over simple master-slave replication because each set of attributes are sent to a different buddy pair.

Serialized Item Consumption when each client's request is for a specific object. The buddy system greatly improves latency over simple master-slave replication because each request is sent to a different buddy pair. 
Table 1. Data Structures

(a) Cluster List

\begin{tabular}{|c|c|}
\hline Cluster & IP \\
\hline \hline 1 & 192.168 .1 .1 \\
\hline 2 & 192.168 .1 .2 \\
\hline 3 & 192.168 .1 .3 \\
\hline
\end{tabular}

(c) Cluster Object Table

\begin{tabular}{|c|c|c|}
\hline Cluster & Object & Version \\
\hline \hline 1 & $\mathrm{~A}$ & 1014 \\
\hline 2 & $\mathrm{~A}$ & 1014 \\
\hline 3 & $\mathrm{~A}$ & 1012 \\
\hline 1 & $\mathrm{~B}$ & 954 \\
\hline 2 & $\mathrm{~B}$ & 954 \\
\hline 3 & $\mathrm{~B}$ & 954 \\
\hline 1 & $\mathrm{C}$ & 2054 \\
\hline 2 & $\mathrm{C}$ & 2054 \\
\hline 3 & $\mathrm{C}$ & 2054 \\
\hline \multicolumn{3}{|c}{} \\
\end{tabular}

Analysis of lost updates in the presence of failures: In each proposed lazy replication scenario, there is one master for a particular data item. After a transaction has committed, there is a period of time where there is the vulnerability that a lost update can occur if hardware hosting the master replica fails before the lazy update propagation is initiated.

The window of vulnerability is not present using eager replication because the updating transaction cannot commit until all other replicas are also updated. However, the update cost of eager update propagation is high, and, therefore, it is not used frequently.

Using the buddy system, we increase durability over lazy replication while maintaining efficient performance. The weakest point of the buddy system is the durability of the dispatcher. If the dispatcher fails, the data structures may get lost and recovery activities must be performed. For this reason, we require that the data structures maintained by the dispatcher are backed by traditional database durability support.

\subsection{Empirical results}

We tested the performance of our Buddy-system against the lazy and eager replica update protocols. We also considered two possible communication architectures: synchronous and asynchronous communication. Using asynchronous communication, the client sends a request and waits for a response to be sent asynchronously. In synchronous communication the client waits until the response is received. The major difference in these two methods is how the enqueue process is handled when the dispatcher cannot fulfill the request with the current state of the clusters. Figure 2 shows the empirical data from an implementation using synchronous requests. The dispatcher is written in Java EE using a Tomcat servlet container. The dispatcher uses class attributes to share hash tables,

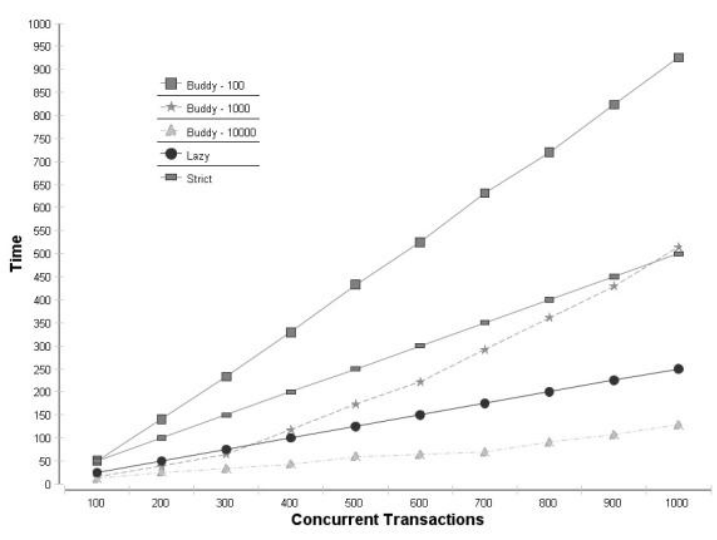

Figure 2. Performance Results

the internal data structures, across all request threads. Each cluster is also implemented in Java EE using a Tomcat servlet container. A separate MYSQL database is used by each cluster in serializable isolation. The cluster uses a JDBC connection pool communicating to its individual database.

A dataset with different sizes was generated with each transaction randomly selecting two items to read and one item to write. Buddy-100, Buddy1000 and Buddy-10000 represent the performance of the Buddy algorithm with a dataset size of 100, 1000, and 10000 items, respectively. The same transactions were run against a single master cluster system with lazy replication and a two cluster system with strict replication. Figure 2 shows that once the dataset size grew to 10,000 items the performance of the Buddy algorithm allowed the system to exceed the performance of lazy replication while increasing durability.

The severe performance penalty observed with small datasets is the result of the enqueue process and the overhead of selecting buddies. Our ongoing work aims to improve the buddy selection algorithm and to reduce the number of transactions that cannot be processed concurrently. Also, in the current implementation the dispatcher stores the version data structures in memory. Our future implementation stores these tables in secondary storage to increase redundancy and durability of the dispatchers' data.

\section{Anonymous resource consumption}

To reduce the performance penalty observed in our initial implementation we modified our solution to be able to guarantee that resource capacity is enforced in a way that we can distribute simultaneous writes to different clusters.

The problem of anonymous resource consumption is a problem that has driven many system designers away from using a RDBMS because of the way resource contention is handled in traditional database system. The popular locking 
mechanism, used to ensure serializability, does not allow for an efficient standard solution to the anonymous resource consumption problem.

To solve the problem of anonymous resource consumption, we introduce a new constraint. Allowing the dispatcher to keep a capacity value for each resource allows the buddy selection algorithm to treat updates to an item as separate writes. The original dispatcher Algorithm 1 distinguished between writes and updates by the data item version in the versions table. If an in-progress version was found it was considered an update otherwise it was considered a write. Our new dispatcher Algorithm checks the capacity of the requested resource. If there is available capacity the update is converted to a write by using an initialization version number instead of the actual version.

\subsection{Dispatcher data structures}

The dispatcher maintains the three original data structures, Table 1.a, b, c from the Buddy System for processing the algorithms, along with a new structure Table 1.d to represent resource availability. The Object Capacity Table contains the name of the data items and their capacities.

\subsection{Analysis of the Buddy System on resource consumption}

Figure 4 shows the performance of the new Buddy System algorithm compared against the lazy and eager replica update protocols. The new algorithm, using the capacity constraints, outperforms lazy replication on all types of resource consumption. Using the Buddy System on our earlier example transaction improves the availability of the TRS by allowing more clusters to participate in the transaction through the use of different masters for each seating location. The TRS also has a guarantee of consistency and durability.

\section{Coarse-grained web services}

In the previous sections we considered web services as fine-grained CRUD services similar to a database transaction. In this section, we target coarse-grained web services, where the only information the dispatcher has at runtime is the input and output parameters of the web service.

\subsection{Example transaction expanded}

Consider a Ticket Reservation System (TRS) presented in Section 3. Clients may want to select a specific seat for the popular concert as a step in the ticket reservation transaction. In Figure 3, we show an implementation of this functionality.
Upon receiving a client request, the web application needs to communicate with a set of web services to gather the data required to render the current seating map and allow the limited resource (the seats) to be consumed. The seating map needs to convey several pieces of information to the user, including:

- Visual representation of sold and available seats

- $\quad$ Pricing for the current user

- Performance details.

After the users have selected a set of seats they would like to purchase, a web service is invoked that finalizes the seat reservation transaction. In Figure 3 , we show the web services of the TRS.

- GetSession - This web service retrieves session state based on a unique session id.

- LoginAnonymous - This web service logs a user in so they can retrieve credentials for pricing and seating location availability. If the session does not have a logged in credential, it gives the user the "anonymous" credentials.

- GetZones - This web service retrieves the zone information for the space where the event takes place. This information is used to allow a user to navigate between zone information. This information does not typically change after a ticketed event has been setup.

- GetSeats - This web service retrieves seating location for the current or default zone. Seating information is composed of a set of seats that have attributes for section, row and seat numbers. This information does not typically change after a performance has been setup.

- GetSeatState - This web service retrieves state information for all the seats in the zone. This information changes when any seat is consumed by another user.

- GetPerformanceDetails - This web service retrieves program details for the performance that is being sold. This information does not typically change after a performance has been setup.

- ReserveSeats - This web service consumes the limited resource and changes the state of the previous GetSeatState web service.

The number of requests from clients may vary drastically over time. During normal operations an organization may only have a few concurrent requests. When a popular event goes on sale, this number could rise to tens of thousands of requests. If several events go on sale at the same time then the 
services may need to handle hundreds of thousands of simultaneous requests.

The popular way to handle unknown workload is by manually partitioning the data across different servers. For example each event could have its own ReserveSeats. However, this solution does not scale well as new hardware would be needed to handle higher levels of event concurrency and reduces consistency in the process.

\subsection{UML semantics}

Additional semantics for the coarse-grained web services can be acquired from integration of the matching UML Activity and Class diagrams. UML provides an extensibility mechanism that allows a designer to add new semantics to a model. A stereotype is one of three types of extensibility mechanisms in the UML that allows a designer to extend the vocabulary of UML in order to represent new model elements [4]. Traditionally these semantics were used by the programmer manually and translated into the program code in a hard coded fashion.

Read and write semantics. Figure 3 is an activity diagram with two stereotypes used to model web services that are read-only and web services that write and update data as part of the process. In the example the ReserveSeats services modifies data as part of its process and all other services just read data as part of their process.

Element unique identifier semantics. Each web service in the Activity diagram has a matching UML Class diagram that shows the structure of the input and output messages. Note that, the same data can be retrieved from the WSDL [5] message types. In this work we use the data from the UML diagram.

An attribute level stereotype $\langle\langle\mathrm{PK}\rangle\rangle$ is used to represent the unique identifier combination of the resources. For example in the GetSeatStatus web service, an individual seats status can be uniquely identified in the response by the attribute set \{Performance, Zone, SeatId\}. The unique identifier also used by the ReserveSeatsRequest service as the input parameter.

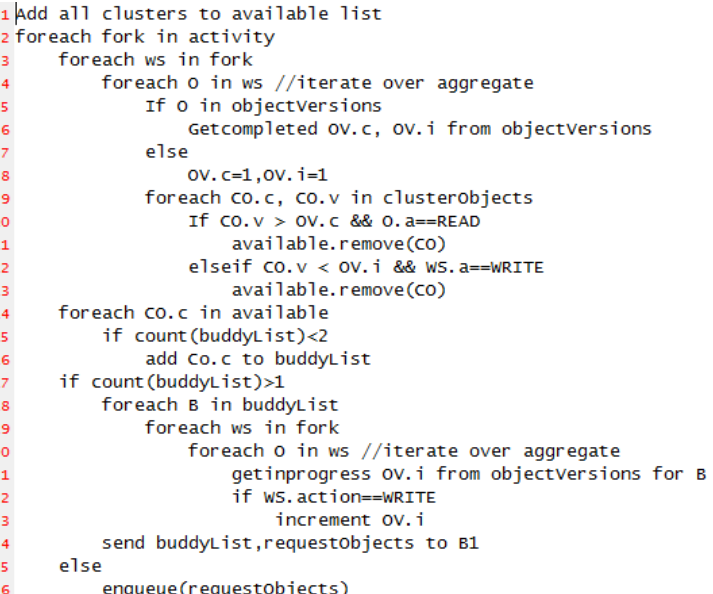

\section{Algorithm 2. Coarse-Grained Buddy Selection}

\subsection{Parallel scheduling semantics}

The UML Activity diagram (Figure 3) also provides us with the semantics required to know which services can be called in parallel. The GetSession and LoginAnonymous services are required to be called before the remaining services because they change required state used by the later service. This data is exported to an XMI file. The $\mathrm{XMI}$ is a standard XML layout to represent the UML diagram. The web services form a directed acyclic graph (DAG). A fork node separates a flow into several parallel control flows. A join node joins several parallel control flows once a token has arrived from each flow. The fork, join, and each web service are represented as ownerMember XML elements with a unique identifier. Each path leads to the join node where the dispatcher waits for all paths to complete. A breadth first search algorithm that uses parallel traversal is used to follow all the parallel paths in the fork.

\subsection{Buddy system changes to handle coarse- grained services}

The original buddy system received a single packet of the fine-grained operations in the transaction. In normal web service operations, a client application is responsible for calling each operation individually. The Dispatcher Service Request Algorithm (Algorithm 1) needs visibility into all operations of the transaction at a single point

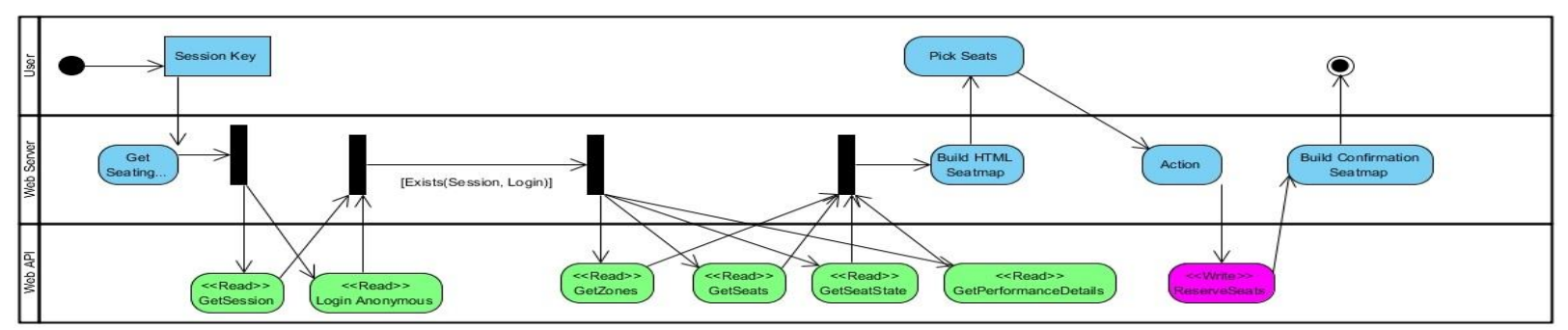

Figure 3. Activity diagram for ticketing seat selection 
in time. To facilitate this visibility, the client sends all requests as a batch and the dispatcher sequences the calls based on the semantics from the XMI data.

Buddy selection algorithm. Algorithm 2 is an updated buddy selection algorithm to select the appropriate pair of web services to perform the transaction. The algorithm iterates over the forks in the activity diagram to service the items that can be done in parallel. A fork is a point in the activity diagram where the flow is split and can run in parallel. Within each fork the algorithm iterates over each web service and flattens the class diagram to get one instance per aggregation. Each instance is then iterated over and its current version is checked in the version tables to determine its current version. The algorithm then determines eligible buddies that can service the batch of web service requests and randomly chooses two to do so.

Theorem 2: The Coarse-Grained Buddy Algorithm (Algorithm 2) guarantees one-copy serializability.

Proof: Our proof is based on the following claim: Let $\mathrm{H}$ be a history over a set of transactions $\mathrm{T}$, such that each transaction $\mathrm{T}_{\mathrm{i}} ;\{\mathrm{i}=1, \ldots, \mathrm{n}\}$ is made up of a set of web services $\mathrm{WS}_{\mathrm{i}}$. Each web service is made up with a setup of operations that are either read $r_{i}(d)$ or write $w_{i}(d)$ operations on data item $d . H$ is one-copy serializable if the following three conditions hold:

1. Each request (transaction) is an atomic transaction

2. Concurrent writes on the same data item are sent to the same cluster, and

3. Each cluster guarantees serializable transaction history on their local database.

To show that the claim holds, assume, by contradiction that $\mathrm{H}$ is not one-copy serializable. Then, there must exist a cycle among the committed transactions in the serialization graph of $\mathrm{H}$. Let $\mathrm{T}_{\mathrm{i}}$ and $T_{j}$ be the two transactions responsible for the cycle. Since Theorem 1 holds, and Algorithm 2 maps from the coarse-grained web services to the finegrained CURD operations of Algorithm 1, then the serialization graph cannot contain a cycle.

\subsection{Coarse-grained implementation}

We used Visual Paradigm ${ }^{\text {TM }}$ for the UML diagrams and exported the diagrams to XMI using the built in export functionality. On startup the dispatcher created a precedence graph based on the semantics of the XMI data. We ran the results against a concurrent load of users and measured the time until completion. In Figure 4, we show the results where we compare three different modes of operation against the time it takes for blocks of users to complete the requests. The users were tested in blocks of 50 and tested against three different

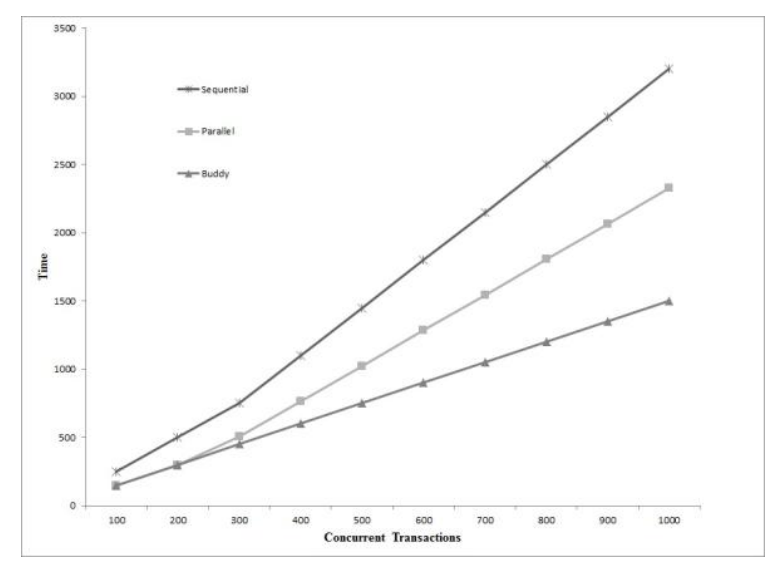

Figure 4. Empirical results for CoarseGrained Implementation

architectures, where each web service was called sequentially using no UML semantic data, in parallel using the semantic data from the UML Activity diagram, and distributed using the semantic data from both the activity and the class diagrams.

Transaction details. In the example transaction the web application sends the set of web service requests \{GetSession, LoginAnonymous, GetZones, GetSeats, GetSeatState, GetPerformanceDetails \} to the dispatcher. In sequential mode the services would be scheduled in a sequence on the same web service box.

Using the semantic data from the UML Activity diagram Figure 3, the dispatcher determines that a sequence of two subsets is required:

1. $\{$ GetSession, LoginAnonymous

2. \{GetZones, GetSeats, GetSeatState, GetPerformanceDetails \}

Using these semantics, the services in the same subsets can be scheduled in parallel for an improvement in performance over the original sequential schedule. Algorithm 2 allows the dispatcher to take this a step further by looping through fine-grained objects read or written by the individual web service. This information is gained from two places:

1. The stereotype in the UML activity diagram (Figure 3) indicates the read and write activities.

2. The individual items from the UML class diagrams represent the fine-grained items.

The $\langle<\mathrm{PK}\rangle>$ stereotype in the UML class diagrams allows us to uniquely identify each tuple in the fine- grained operations. Once these semantics have been identified the original buddy algorithm (Algorithm 1) can be implemented on the coarsegrained services. 
Figure 4 shows the performance results of the implementation where the additional semantics gained from the UML data allows the buddy system to almost double the availability of the original sequential schedule.

\section{Related work}

Most of the distributed database research ignores resource consumption issues and assumes traditional locking mechanisms. Julian Jang et al. [6], investigate ways to provide non-locking resource consumption for a longer duration than the transaction to avoid holding locks. Unfortunately this approach sacrifices ACID guarantees that are provided by traditional RDMS. One of the current application areas for replicated databases is web services applications. Lou and Yang [7] study the two primary replica update protocols in the context of web services. The authors state that eager replication has a problem of increasing latency as the number of replicas increases. This increasing latency diminishes the availability gains from introducing replicas. Most commercial implementations use lazyreplication because of its efficiency and scalability. Lazy replication methods are also more partition tolerant than eager replication methods. However, lazy-replication protocols require additional considerations to ensure consistency. Research has been conducted for decades on strict and lazy replication in RDMS. Recent research can be grouped into one of three goals: 1.) trying to increase availability for strict replication, 2.) trying to increase consistency for lazy replication, and 3.) attempting to use a hybrid approach.

\subsection{Higher availability with strict replication}

Several methods have been developed to ensure mutual consistency in replicated databases. The aim of these methods is to eventually provide one-copy serializability (1SR). Transactions on traditional replicated databases are based on reading any copy and writing (updating) all copies of data items. Based on the time of the update propagation, two main approaches have been proposed. Approaches that update all replicas before the transaction can commit are called eager update propagation protocols; approaches that allow the propagation of the update after the transaction is committed are called lazy update propagation. While eager update propagation guarantees mutual consistency among the replicas, this approach is not scalable. Lazy update propagation is efficient but it may result in violation of mutual consistency. During the last decade, several methods have been proposed to ensure mutual consistency in the presence of lazy update propagation (see [8]for an overview.) More recently,
Snapshot Isolation (SI) $[9,10]$ has been proposed to provide concurrency control in replicated databases. The aim of this approach is to provide global onecopy serializability using SI at each replica. The advantage is that SI provides scalability and is supported by most database management systems.

\subsection{Higher consistency in lazy replication}

Breitbart and Korth [11], and Daudjee et al. [12] propose frameworks for master-slave, lazyreplication updates that provide consistency guarantees. These approaches are based on requiring all writes to be performed on the master replica. Updates are propagated to the other sites after the updating transaction is committed. Their framework provides a distributed serializable schedule where the ordering of updates is not guaranteed.

The approach proposed by Daudjee et al. provides multi-version serializability where different versions of data can be returned for read requests during the period that replication has not completed.

\subsection{Hybrid approach}

Jajodia and Mutchler [13], and Long et al. [14], define forms of hybrid replication that reduce the requirement that all replicas participate in eager update propagation. The proposed methods aim to increase availability in the presence of network isolations or hardware failures. Both approaches have limited scalability because they require a majority of replicas to participate in eager update propagation. Most recently, Garcia-Munos et al. [15] proposed a hybrid replication protocol that can be configured to behave as eager or lazy update propagation protocol. The authors provide empirical data and show that their protocol provides scalability and reduces communication cost over other hybrid update protocols. In addition to academic research, several database management systems have been developed that support some form of replicated data management. For example, Lakshman and Malik [16] describe a hybrid system, called Cassandra, which was built by Facebook to handle their inbox search. Cassandra allows a configuration parameter that controls the number of nodes that must be updated synchronously. The Cassandra system can be configured so nodes chosen for synchronous inclusion cross data center boundaries to increase durability and availability.

\section{Conclusion}

In this paper we propose an extension to the lazy replica update propagation methods. Our solution is based on using an application-layer dispatcher to select two web service clusters, called buddies, to simultaneously perform a transaction. The buddy 
selection is based on the data items and the operations of the transactions, the data versions available, and the network characteristics of the WS farm.

We show that although our solution can be viewed as a special case of hybrid update propagation method, it provides several advantages over existing solutions.

First, our approach provides the scalability required by modern applications, such as web services, and is suitable for the architectures and technologies implementing these applications. The buddy selection algorithm supports dynamic masterslave site selection for data items, ensures correct transaction execution, and aids load-balancing among the replicas. Finally, incorporating network specific characteristics, such as distance and bandwidth, reduces latency observed by the client. Our approach has a similar message complexity compared to a traditional lazy master-slave replication using group communication and less message complexity compared to a lazy master-slave replication without group communication.

We further extend the buddy system to handle coarse-grained web services. Our solution is based on extending UML specifications with stereotypes to embed CRUD, Parallel and data element semantics into the model. The dispatcher can then extract the semantics from the model and distribute the requests to clusters as it did with the fine-grained web service. Each individual transaction is applied to a pair of clusters synchronously allowing enforcement of consistency guarantees and durability. There are two limitations of our work we plan to address in the future. First, we are relying on the back end database for constraint guarantees. This limits the granularity of transaction parallelism. Second, we require a transaction to be sent in a single request to the dispatcher. We plan to extend the buddy system to allow the dispatcher to receive WS-Atomic and WSBusinessActivity transactional requests that can span a single request.

\section{References}

[1] S. Gilbert and N. Lynch, "Brewer's conjecture and the feasibility of consistent, available, partition-tolerant web services," SIGACT News, vol. 33, pp. 51-59, 2002.

[2] D. Abadi, "Consistency tradeoffs in modern distributed database system design: Cap is only part of the story," Computer, vol. 45, pp. 37-42, 2012.

[3] A. Fekete, S. N. Goldrei and J. P. Asenjo, "Quantifying isolation anomalies," in Proceedings of the VLDB Endowment, 2009.

[4] O. M. Group, "Unified Modeling Language: Supersturcture," 0502 2007. [Online]. Available: http://www.omg.org/spec/UML/2.1.1/. [Accessed 08
01 2013].

[5] E. Christensen, F. Curbera, G. Meredith and S. Weerawarana, "Web service definition language (WSDL)," 2001. [Online]. Available: http://www.w3.org/TR/wsdl.

[6] J. Jang, A. Fekete and P. Greenfield, "Delivering promises for web," in Web Services, IEEE International Conference on, 2007.

[7] M. Y. Lou and C. S. Yang, "Constructing zero-loss web services," INFOCOM, pp. 1781-1790, 2001.

[8] M. T. Ozsu and P. Valduriez, Principles of Distributed Database Systems, 3rd ed., Springer, 2011.

[9] Y. Lin, B. Kemme, M. Patino Martinez and R. Jimenez-Peris, "Middleware based data replication providing snapshot isolation," in Proceedings of the 2005 ACM SIGMOD international conference on Management of data, ser. SIGMOD '05, New York, NY, 2005.

[10] H. Jung, H. Han, A. Fekete and U. Rhm, "Serializable snapshot isolation," PVLDB, pp. 783-794, 2011.

[11] Y. Breitbart and H. F. Korth, "Replication and consistency: being lazy helps sometimes," Proceedings of the sixteenth ACM SIGACTSIGMOD-SIGART symposium on Principles of database systems, ser. PODS '97, pp. 173-184, 1997.

[12] K. Daudjee and K. Salem, "Lazy database replication with ordering," in Data Engineering, International Conference on, 2004.

[13] S. Jajodia and D. Mutchler, "A hybrid replica control algorithm combin-ing static and dynamic voting," IEEE Transactions on Knowledge and Data Engineering, vol. 1, pp. 459-469, 1989.

[14] D. Long, J. Carroll and K. Stewart, "Estimating the reliability of," IEEE Transactions on, vol. 38, pp. 1691-1702, 1989.

[15] L. Irun-Briz, F. Castro-Company, A. Garcia-Nevia, A. Calero-Monteagudo and F. D. Munoz-Escoi, "Lazy recovery in a hybrid database replication protocol," in In Proc. of XII Jornadas de Concurrencia y Sistemas Distribuidos, 2005.

[16] A. Lakshman and P. Malik, "Cassandra: a decentralized structured," SIGOPS Oper. Syst. Rev., vol. 44, pp. 35-40, 2010.

[17] A. Olmsted and C. Farkas, "The cost of increased transactional correctness and durability in distributed databases," in 13th International Conference on Information Reuse and, Los Vegas, NV, 2012.

[18] A. Olmsted and C. Farkas, "High Volume Web Service Resource Consumption," in Internet Technology and Secured Transactions, 2012. ICITST 2012, London, UK, 2012. 